\title{
Kalirin, a Key Player in Synapse Formation, Is Implicated in Human Diseases
}

\author{
Prashant Mandela and Xin-Ming Ma \\ Department of Neuroscience, University of Connecticut Health Center, Farmington, CT 06030, USA \\ Correspondence should be addressed to Xin-Ming Ma, ma@nso.uchc.edu \\ Received 30 November 2011; Accepted 13 January 2012 \\ Academic Editor: Irina Nikonenko
}

Copyright ( $) 2012$ P. Mandela and X.-M. Ma. This is an open access article distributed under the Creative Commons Attribution License, which permits unrestricted use, distribution, and reproduction in any medium, provided the original work is properly cited.

\begin{abstract}
Synapse formation is considered to be crucial for learning and memory. Understanding the underlying molecular mechanisms of synapse formation is a key to understanding learning and memory. Kalirin-7, a major isoform of Kalirin in adult rodent brain, is an essential component of mature excitatory synapses. Kalirin-7 interacts with multiple PDZ-domain-containing proteins including PSD95, spinophilin, and GluR1 through its PDZ-binding motif. In cultured hippocampal/cortical neurons, overexpression of Kalirin-7 increases spine density and spine size whereas reduction of endogenous Kalirin-7 expression decreases synapse number, and spine density. In Kalirin-7 knockout mice, spine length, synapse number, and postsynaptic density (PSD) size are decreased in hippocampal CA1 pyramidal neurons; these morphological alterations are accompanied by a deficiency in long-term potentiation (LTP) and a decreased spontaneous excitatory postsynaptic current (sEPSC) frequency. Human Kalirin-7, also known as Duo or Huntingtin-associated protein-interacting protein (HAPIP), is equivalent to rat Kalirin-7. Recent studies show that Kalirin is relevant to many human diseases such as Huntington's Disease, Alzheimer's Disease, ischemic stroke, schizophrenia, depression, and cocaine addiction. This paper summarizes our recent understanding of Kalirin function.
\end{abstract}

\section{Kalirin Is a Rho Guanine Nucleotide Exchange Factor (GEF)}

Kalirin was discovered 15 years ago as a novel protein that interacts with the cytosolic carboxyl-terminal of peptidylglycine $\alpha$-amidating monooxygenase (PAM), an integral membrane peptide processing enzyme [1]. We have made significant progress in understanding the functions of Kalirin; like the many other Rho-GEFs encoded in mammalian genomes, Kalirin promotes the exchange of GDP for GTP and thus stimulates the activity of specific Rho GTPases $[2,3]$. Rho GTPases that regulate multiple cellular processes play a key role in transducing signals from extracellular stimuli to the intracellular pathways that play a pivotal role in the formation of dendritic spines and synaptic development [4-6].

\section{Multiple Kalirin Isoforms}

The mouse Kalirin gene (Kalrn) consists of 65 exons spanning $>650 \mathrm{~kb}$ of the genome; the presence of multiple promoters and transcriptional start sites enables the production of multiple functional isoforms of Kalirin [7-9]. Each Kalirin isoform is composed of a unique collection of domains (Figure 1). Major Kalirin isoforms including Kalirin-7, -9 , and -12 are generated through the use of alternative $3^{\prime}$ exons [8]. The major isoforms share some common features including nine spectrin-like repeats, the GEF1 domain and the Sec14p domain. Sec14p domains facilitate lipid interactions and cellular localization. The nine spectrin-like repeat regions that follow the Sec14p domain have been shown to interact with many proteins including disrupted in schizophrenia 1 (DISC1) [10], peptidylglycine $\alpha$-amidating monooxygenase (PAM) [1], inducible nitric oxide synthase (iNOS) [11], Huntingtin-associated protein 1 (HAP1) [12], and Arf6 (ADP-ribosylation factor 6) [13]. Kalirin-12 is the longest isoform and contains additional domains that include GEF2, an immunoglobulinlike (Ig) domain, a fibronectin III (FnIII) domain, and a serine/threonine protein kinase domain that is followed by a short, unique carboxyl-terminus [7, 14]. Kalirin-12 is found 


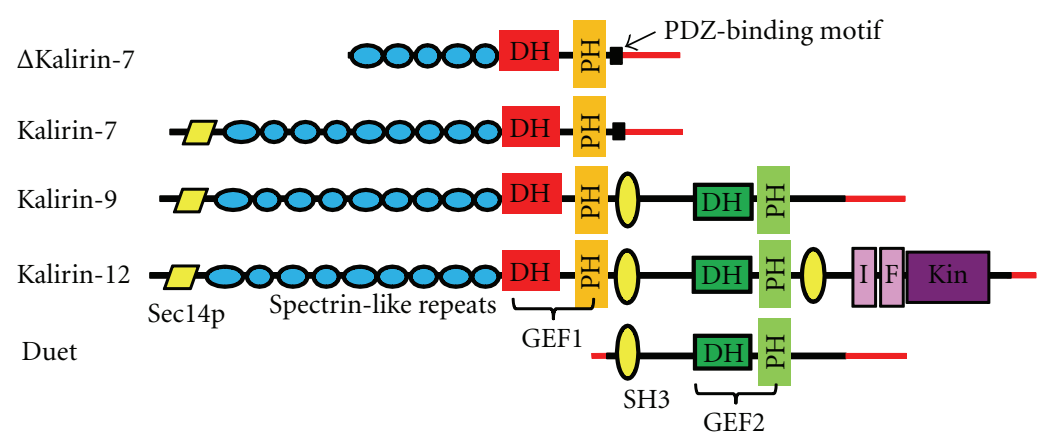

FIGURE 1: Major Kalirin isoforms. Alternative splicing generates different isoforms of Kalirin. DH: Dbl homology; PH: pleckstrin homology; GEF1: guanine nucleotide exchange factor 1; SH3: Src homology domain; GEF2: guanine nucleotide exchange factor 2; I: immunoglobulinlike; F: fibronectin III-like; Kin: kinase domain; red lines, unique $5^{\prime}$ - and $3^{\prime}$-untranslated regions.

in the growth cones of immature neurons (Figure 2) and dendritic spines of mature cultured hippocampal neurons, suggesting a role for Kalirin-12 in axon outgrowth and synaptic plasticity. Interaction of the Ig-FnIII region unique to Kalirin-12 with the GTPase domain of dynamin may facilitate the coordination of endocytic trafficking and changes in the actin cytoskeleton [15]. Endogenous Kalirin9 is localized to neurites and growth cones, and expression of exogenous Kalirin-9 induces longer neurites and altered neuronal morphology in cultured cortical neurons [16]. The functions of Kalirin-9 and Kalirin-12 in neurons remain to be elucidated. The $\Delta$ Kalirin-7 (also referred as Kalirin5) isoform is generated using a different promoter, and translation initiation begins at the start of spectrin-like repeat 5 , producing an isoform with only 5 spectrin-like repeats. Overexpression of $\Delta$ Kalirin-7 results in an increase in spine size, but not spine density, in cultured cortical neurons [17]. Duet, an isoform of Kalirin that begins just before the second GEF domain and continues through the unique C-terminal of Kalirin-12, uses a third promoter [7]. Duet was discovered as a protein homologous to the catalytic domain of death associated protein kinase and is localized to actin-associated cytoskeletal elements, suggesting the involvement of Duet in cytoskeleton-dependent functions [18].

\section{Tissue Expression}

Kalirin expression is detectable in a wide array of adult tissues including neurons, endocrine cells, liver, muscle, and heart $[2,20,21]$. In addition, developmentally regulated, tissue specific-Kalirin isoform expression is evident. Kalirin9 and -12 are highly expressed in neuronal tissue during embryonic development, while in the adult brain expression of each is drastically decreased; a concomitant increase in Kalirin-7 expression occurs [8, 20, 22]. Kalirin-7 expression is largely limited to neurons of the central nervous system; its levels are extremely low at birth (postnatal days 17) and begin to increase markedly at postnatal day 14 , which coincides with the onset of maximum synaptogenesis $[22,23]$.

\section{Kalirin-7 Is the Major Kalirin Isoform in Adult Brain}

Kalirin-7 is the most abundant Kalirin isoform in the adult rodent brain and is exclusively localized to the postsynaptic side of excitatory synapses $[19,22,39,40]$. Kalirin-7 is a multifaceted molecule containing domains that interact with a wide array of molecular machinery. The Sec14p domain located at the N-terminus interacts with phosphatidylinositol-3-phosphate and plays a key role in Kalirin-7-mediated spine morphogenesis (Ma et al., unpublished). The C-terminus of Kalirin-7 contains a unique PDZbinding motif through which Kalirin-7 interacts with PDZdomain-containing proteins including PSD-95, AF-6, and spinophilin [39]. Binding of the NMDA receptor subunit NR2B to the PH domain of Kalirin-7 is important for normal synaptic plasticity [41]. The spectrin-like domains of Kalirin-7 through which it interacts with DISC1, iNOS, PAM, HAP1, and Arf6 play a key role in Kalirin-7-induced synapse formation (Ma et al., unpublished). The nucleotide sequences of human Kalirin-7 (Duo) and rat Kalirin-7 are 91\% identical, and their amino acid sequences are 98\% identical; human Kalirin-7 contains a 27-nucleotide insert not found in rat Kalirin-7, located at the end of the region encoding the seventh spectrin repeat $[8,12]$.

\section{Kalirin-7 Contains Multiple Phosphorylation Sites}

Phosphorylation of Kalirin-7 has recently been shown to be a pivotal mechanism mediating Kalirin-7-induced spine formation and synaptic plasticity [42-44]. Purified PKA, PKC, CaMKII, Cdk5, and Fyn each phosphorylate purified Kalirin-7 [45]. Kalirin-7 is extensively phosphorylated in vivo. The phosphorylation sites identified in vitro using purified CaMKII, PKA, PKC, or CKII, identified only 5 of the 22 sites that undergo phosphorylation in cells or tissue. These findings emphasize a critical role for additional protein kinases and the importance of cellular localization in the phosphorylation of Kalirin-7 [45]. Densely distributed 

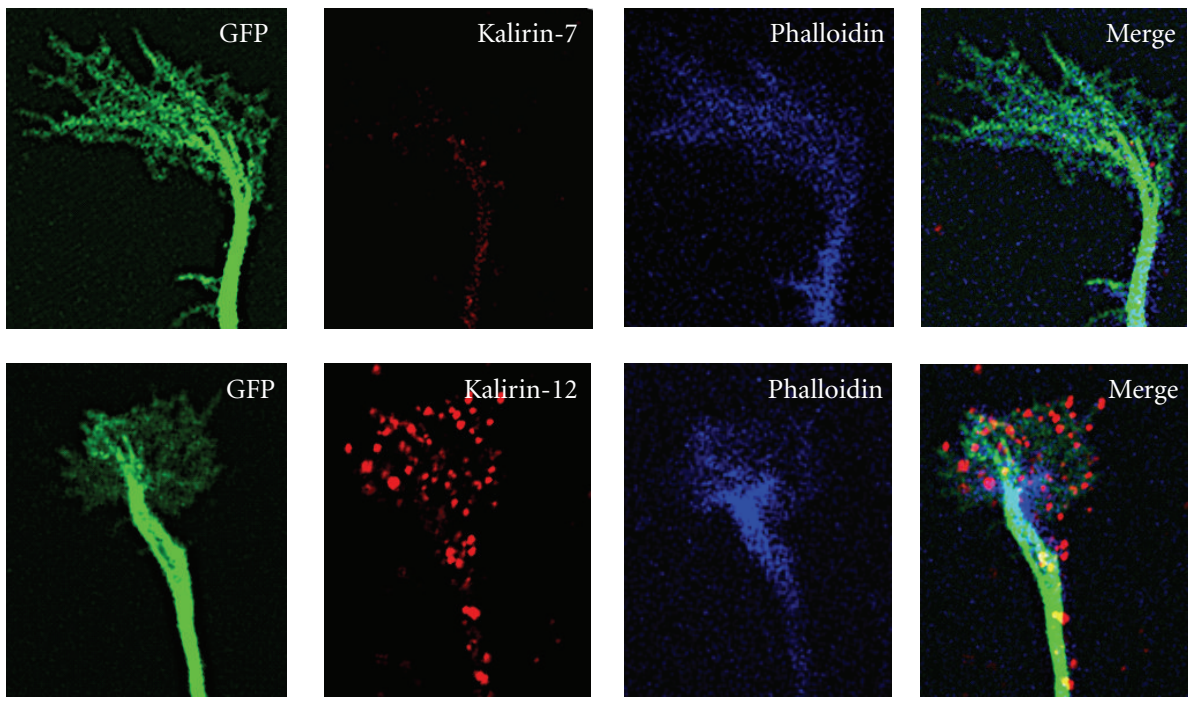

Figure 2: Kalirin-12, not Kalirin-7, is localized to the growth cone of hippocampal neurons. Primary cultures of hippocampal neurons prepared at embryonic day 20 (E20) were transfected with a vector encoding GFP on the day of culture preparation as described [19]. On day 3 after transfection, cultures were fixed for staining of filamentous actin with Alexa Fluor 633 Phalloidin (Life Technologies) and endogenous Kalirin-7 or Kalirin-12 with isoform-specific rabbit antibodies. Rabbit antibodies were visualized using Cy3 donkey anti-rabbit IgG (The Jackson Laboratory). Images were collected with a Zeiss confocal microscope LSM510.

phosphorylation sites have been identified in the spectrinlike repeat region, many lying on the fourth and fifth repeats. Their phosphorylation state could influence on a wide array of protein-protein interactions that involve this region. Since the first four spectrin-like repeats are absent in $\Delta$ Kalirin-7 (Figure 1), the phosphorylation sites in the missing region could play a key role in the differences in spine size and density associated with expression of full length Kalirin-7 versus $\Delta$ Kalirin-7. Overexpression of exogenous Kalirin-7 results in an increase in both spine density and spine size while overexpression of $\Delta$ Kalirin-7 only increases spine size without altering spine density [17]. Although phosphorylation sites that regulate GEF activity as assessed in intact cells have been identified, a relationship between phosphorylation state and GEF activity has not yet been demonstrated using purified proteins.

\section{Kalirin-7 Plays a Key Role in Spine/Synapse Formation In Vitro}

Kalirin-7 expression is limited to neurons in the CNS [22, 30, 46]. Immunostaining in cultured hippocampal and cortical neurons demonstrates that Kalirin-7 clusters are apposed to glutamate-transporter-1-(Vglut1-) positive clusters, a marker for excitatory presynaptic terminals [19, 47, 48]. When cultured neurons are fixed with cold methanol, it can be seen that the Kalirin-7 positive clusters consistently overlap clusters positive for PSD95, NMDA receptor subunits NR1 and NR2B, or AMPA receptor subunits GluR1 and GluR2; overlap is apparent in both dendritic spines and in the dendritic shaft $[19,40]$. In contrast, Kalirin-7 clusters neither align with GAD65-positive clusters, a marker for inhibitory presynaptic terminals, nor with $\mathrm{GABA}_{\mathrm{A}}$ receptor positive clusters, a marker for inhibitory postsynaptic endings [19]. These findings lead to the conclusion that Kalirin-7 is localized almost entirely to postsynaptic excitatory terminals.

Regulation of Kalirin-7 expression by synaptic activity in hippocampal neurons suggests that Kalirin-7 plays a pivotal role in the regulation of excitatory synapse formation and signaling [40, 42]. Overexpression of Kalirin-7 causes an increase in dendritic spine density, spine size, and synapse number, while reduction of endogenous Kalirin-7 levels leads to a reduction in spine density, spine size, and synapse number in cultured hippocampal and cortical neurons [19, 22, 39] (Figure 3). Interestingly, overexpression of Kalirin-7 induces spine formation in spine-free hippocampal interneurons [19] and a recent study reports an important role of Kalirin-7 in regulating dendrite growth in cortical interneurons [44]. A delicate balance between synaptic excitation and inhibition is critical for maintaining normal circuits in the CNS, and interneurons play an essential role in regulating local circuit excitability [49]. Understanding Kalirin-7 function in interneurons may increase our understanding of neurological diseases such as epilepsy, bipolar disorder, schizophrenia, autism, and Alzheimer's Disease, which are related to disruption of GABAergic interneuron development [50-53].

\section{Kalirin-7 Plays a Key Role in Spine/Synapse Formation In Vivo}

Two Kalrn knockout mouse models have been developed: the Kalirin-7 knockout mouse (Kalirin-7 ${ }^{\mathrm{KO}}$ ) [54] and the GEF1 Kalirin knockout mouse (Kalirin GEF1-KO) [55]. Kalirin-7 KO mice, in which the terminal exon unique to Kalirin-7 was deleted, grow and reproduce normally. Hippocampal CA1 


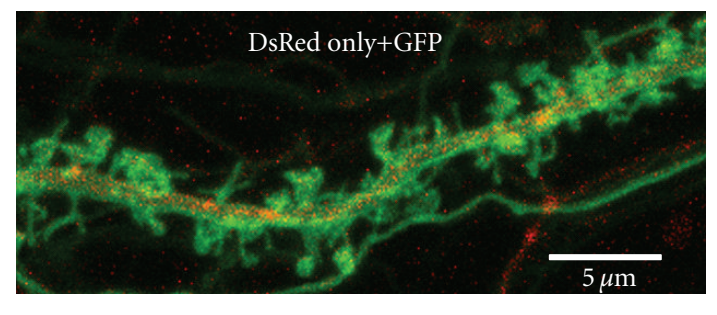

(a)

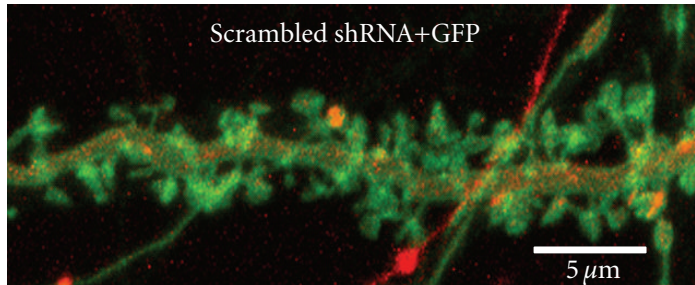

(b)

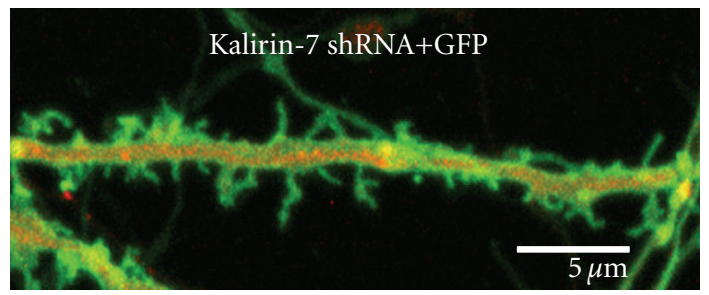

(c)

Figure 3: Expression of Kalirin-7 shRNA causes a reduction in spine density and spine size in cultured hippocampal neurons. Cultured hippocampal neurons prepared at E20 were transfected with vector encoding a membrane-tethered version of GFP (pmGFP) alone [(a), spine density $8.9 \pm 0.8 / 10 \mu \mathrm{m}$ ], pmGFP plus a scrambled shRNA [(b), spine density $9.2 \pm 0.9 / 10 \mu \mathrm{m}$ ], or pmGFP plus pSIREN-Kalirin-7 shRNA [(c), spine density $5.2 \pm 0.6 / 10 \mu \mathrm{m}$ ] on the day of culture preparation. The specificity of pSIREN-Kalirin-7 shRNA was determined previously [19]. DsRed marks transfected neurons expressing the shRNA. Images were collected on day 20 with an LSM510 confocal microscope. Expression of Kalirin-7 shRNA, not scrambled shRNA, caused a 80\% decrease in Kalirin-7 staining (not shown). Spine density was determined using Metamorph (Molecular Devices, Downington, PA) as described [54]. Scale bar $=5 \mu \mathrm{m}$.

pyramidal neurons of Kalirin-7 $7^{\mathrm{KO}}$ mice show a $15 \%$ decrease in spine density and deficits in long-term potentiation (LTP). Morphological alterations in Kalirin- $7^{\mathrm{KO}}$ mice are accompanied by behavioral alterations including decreased anxiety-like behavior in the elevated zero maze and impaired acquisition of a passive avoidance task. Kalirin- $7^{\mathrm{KO}}$ mice exhibit normal behavior in the open field, object recognition, and radial arm maze tasks [54]. PSDs purified from the cortices of Kalirin- $7^{\mathrm{KO}}$ mice show a deficit in Cdk5, a kinase known to phosphorylate Kalirin-7 and play an essential role in Kalirin-7-mediated spine formation and synaptic function $[43,54,56]$. Furthermore, NR2B levels are modestly decreased in PSD preparations from the cortices of Kalirin$7^{\mathrm{KO}}$ animals [54]. This decrease is accompanied by decreased levels of NR2B-dependent NMDA receptor currents in cortical pyramidal neurons [41]. NR2B plays a critical role in LTP induction, dendritic spine formation, different forms of synaptic plasticity, learning, and memory [57-60]. Decreased NR2B levels may partially contribute to decreased spine density and the deficit in LTP induction observed in Kalirin$7^{\mathrm{KO}}$ mice. Importantly, expression of exogenous Kalirin7 in cultured Kalirin- $7^{\mathrm{KO}}$ neurons rescues decreased spine density. These findings show that Kalirin-7 plays an essential role in synaptic structure and function.

The Kalirin ${ }^{\mathrm{GEF} 1-\mathrm{KO}}$ mice were generated by replacing exons 27-28 in the first GEF domain by a neomycin resistance cassette, thus eliminating production of the major Kalirin isoforms; in addition to Kalirin-7, these mice are unable to produce Kalirin-9 and Kalirin-12 [55]. Kalirin GEF1-KO mice show significant morphological deficits including reduced size of both cortex and decreased spine density in pyramidal neurons of the cortex [55]. Interestingly, in
Kalirin ${ }^{\mathrm{GEF} 1-\mathrm{KO}}$ mice the hippocampus is reduced in size but spine density in hippocampal neurons is normal. This reduction in hippocampal size in Kalirin ${ }^{\mathrm{GEF} 1-\mathrm{KO}}$ mice results from neuronal loss since Kalirin is exclusively expressed in pyramidal neurons, granule cells of the dentate gyrus, and interneurons scattered throughout the hippocampus [19, 22, 46, 54]. Both Kalirin- $7^{\mathrm{KO}}$ and Kalirin ${ }^{\mathrm{GEF} 1-\mathrm{KO}}$ mice show impaired hippocampal LTP induction, impaired contextual fear conditioning, and reduced spine density in cultured cortical neurons and normal long-term memory [54, 61]. Kalirin ${ }^{\mathrm{GEF} 1-\mathrm{KO}}$ mice show deficits in working memory and an intact reference memory. However, Kalirin- $7^{\mathrm{KO}}$ and Kalirin ${ }^{\mathrm{GEF} 1-\mathrm{KO}}$ mice exhibit distinct differences in their behavioral phenotype. Kalirin ${ }^{\mathrm{GEF} 1-\mathrm{KO}}$ mice show very high locomotor activity in the open field and a deficit in spatial memory, which are not affected in Kalirin- $7^{\mathrm{KO}}$ mice $[54,55]$. Further comparisons of the phenotypes of the Kalirin GEF1-KO and Kalirin- $7^{\mathrm{KO}}$ mice are needed, along with the generation of additional Kalrn knockout mouse models generated using different knockout strategies.

\section{Kalirin-7 Is Implicated in Cocaine Addiction}

Cocaine addiction is a chronic relapsing neurological disorder associated with severe medical and psychosocial complications [62-66]. The long-lasting nature of cocaine addiction leads to relapse and makes it especially difficult to treat [21]. Repeated cocaine treatments increase dendritic spine density/spine head size and neurite complexity in the brain's reward circuitry such as the medium spiny neurons (MSNs) of the nucleus accumbens (NAc). Our study 
TABLE 1: Kalirin and human diseases.

\begin{tabular}{|c|c|c|c|}
\hline Kalirin isoform & Disease & Physiological relevance & References \\
\hline Kalirin-7 (Duo) & Schizophrenia & $\begin{array}{l}\text { Decreased spine density, decreased Kalirin-7 } \\
\text { mRNA levels in the prefrontal cortex }\end{array}$ & {$[24]$} \\
\hline Kalirin (unknown isoforms) & Schizophrenia & $\begin{array}{l}\text { Kalirin mRNA increases } 2 \text {-fold in the } \\
\text { prefrontal cortex }\end{array}$ & {$[25]$} \\
\hline Kalirin-7 & Schizophrenia & DISC1 regulates spine formation via Kal7-Rac1 & {$[10]$} \\
\hline Kalirin & Schizophrenia & Kalirin is a risk factor for schizophrenia & {$[26]$} \\
\hline Kalirin-7 & Alzheimer's Disease & $\begin{array}{l}\text { Decreased levels of Kalirin-7 mRNA and } \\
\text { protein in hippocampus }\end{array}$ & {$[27,28]$} \\
\hline Kalirin (unknown isoforms) & Animal model of depression & $\begin{array}{l}\text { Decreased Kalirin expression in the prefrontal } \\
\text { cortex and hippocampus. ECT increases } \\
\text { Kalirin expression in hippocampus }\end{array}$ & {$[29,30]$} \\
\hline Kalirin-7 & Animal model of depression & $\begin{array}{l}\text { Decreased Kalirin- } 7 \text { expression in } \\
\text { hippocampus }\end{array}$ & {$[31]$} \\
\hline Kalirin (unknown isoforms) & Animal model of epilepsy & Decreased Kalirin expression in hippocampus & {$[32]$} \\
\hline Kalirin (unknown isoforms) & $\mathrm{ADHD}$ & Unknown & {$[33]$} \\
\hline Kalirin (unknown isoforms) & Stroke & Unknown & {$[34,35]$} \\
\hline Kalirin (unknown isoforms) & Coronary Heart disease & Unknown & {$[36]$} \\
\hline Kalirin (unknown isoforms) & Huntington's disease & $\begin{array}{l}\text { Spectrin-like domains of Kalirin interact with } \\
\text { HAP1 }\end{array}$ & {$[12]$} \\
\hline Kalirin-7 & $\begin{array}{l}\text { Animal model of cocaine } \\
\text { addiction }\end{array}$ & $\begin{array}{l}\text { An essential determinant of dendritic spine } \\
\text { formation following cocaine treatment }\end{array}$ & {$[37,38]$} \\
\hline
\end{tabular}

shows that Kalirin-7 is an essential determinant of dendritic spine formation following cocaine treatment [38]. Kalirin7 is expressed in the MSNs of the NAc, a key area in the brain involved in drug addiction and reward pathways [37]. Chronic cocaine treatment of wild-type mice results in an increase in Kalirin-7 expression in the NAc which is accompanied by an increase in spine density in the MSNs of NAc core $[37,67]$. This cocaine-induced increase in dendritic spine density in the NAc MSNs in wild-type mice is abolished in Kalirin- $7^{\mathrm{KO}}$ mice. Both wild-type and Kalirin- $7^{\mathrm{KO}}$ mice have identical spine densities in the MSNs of the NAc prior to cocaine treatment. These morphological changes could underlie the behavioral variations seen in these mice following cocaine treatment. Chronic cocaine treatment leads to increased locomotor sensitization in Kalirin- $7^{\mathrm{KO}}$ mice compared to wild-type controls [38]. These data suggest Kalirin-7 plays an important role in the mechanism of cocaine addiction, which needs to be addressed in the future.

\section{Kalirin Is Implicated in Human Diseases}

Altered Kalirin expression has been reported in several neuropsychiatric, neurological and cardiovascular diseases as well as animal models of depression, epilepsy and cocaine addiction (Table 1). Genetic analyses have identified KALRN as a major risk factor in stroke and early onset of coronary artery disease [34-36]. Similarly in schizophrenia, decreased dendritic spine density in the prefrontal cortex is reported to correlate with decreased Kalirin mRNA levels [24]. A rare missense mutation in the KALRN gene has been shown to be a genetic risk factor for schizophrenia [26]. The spectrin-like repeat region of Kalirin has also been shown to interact with DISC1, a genetic risk factor for schizophrenia which plays an important role in activity-dependent spine elongation by promoting Kalirin-7/Rac-1 interactions [10, $24,68,69]$. Attention-deficit/hyperactivity disorder (ADHD) is the most common neurobehavioral disorder and the underlying molecular mechanisms of ADHD are largely unknown. Animal models of ADHD are associated with spine loss in striatal MSNs [70] and functional impairments in glutamatergic synaptic transmission in the hippocampus [71]. A genomewide association study of ADHD patients has also implicated alterations in Kalirin expression in ADHD [33]. Dendritic pathology and decreased dendritic spine density are prominent phenomena in early cases of Alzheimer's Disease, which correlate significantly with the progressive decline of mental faculties [72-75]. Alzheimer's Disease patients also show a significant decrease in Kalirin $\mathrm{mRNA}$ and protein expression in the hippocampus without significant changes in other brain regions [28]. This decrease in Kalirin expression has been associated with increased iNOS activity both in hippocampus from Alzheimer's patients and in cultured neuroblastoma cells [27]. These data lead to the hypothesis that lack of Kalirin is associated with the dendritic alterations and substantial decrease in spine density observed in Alzheimer's Disease.

The cause underlying major depression and the neurobiological basis of antidepressant therapy are not clear. Altered synaptic plasticity may play a key role in the pathogenesis 
and treatment of depression [76]. Major depression is associated with spine reductions in the hippocampus [77]. Kalirin expression and spine density in the hippocampus are decreased in the animal models of depression [29, 31, 78]. Kalirin levels and spine density in the hippocampal CA1 pyramidal neurons increase after repeated electroconvulsive treatment (ECT) [30, 79], one of the most effective therapies for depression $[80,81]$. These observations suggest a role for Kalirin in the development of depression. In epileptic patients and in experimental models of epilepsy, there is a marked spine loss, abnormal spine shape, and alteration in dendritic morphology in hippocampal CA1 pyramidal neurons $[82,83]$. Animal models of epilepsy show a marked increase in Kalirin expression in the hippocampus [32], suggesting a role for Kalirin in the neuropathology of human epilepsy. Kalirin interacts with Huntingtin-associated protein 1 (HAP1) [12] and HAP1 dysfunction could contribute to the selective neuropathology in Huntingtin's disease [84]. Huntingtin's disease is characterized by loss of mediumsized spiny neurons in the striatum [85], alterations in spine density, and abnormalities in the size and shape of dendritic spines in striatal MSNs [86-88]. Overexpression of Kalirin-7 caused an increase in spine density while reduced expression of Kalirin-7 resulted in loss of dendritic spines and a decrease in dendritic complexity in the MSNs of striatal slice cultures which mimic in vivo conditions (Ma et al., unpublished). These data raise the possibility that Kalirin-7 may play a role in the neuropathology of Huntington's disease [87]. Finally, Kalirn-7 plays an important role in estrogen-mediated spine/synapse formation in the hippocampus [31, 40]. Regulation of Kalirin-7 by estrogen suggests a role for Kalirin in ovarian hormone-associated cognitive function and menopause-associated disorders [8991]. Taken together, altered dendritic spine morphology and spine density remain the hallmarks of many human neurological and psychiatric disorders. There is a correlation between the levels of Kalirin expression and the pathology of dendritic spines in some psychiatric and neurological disorders. It is important to understand whether Kalirin is a trigger or one of many factors mediating the dendritic spine pathology of these diseases, which may be rescued by altering Kalirin expression/function or targeting downstream signals of Kalirin.

\section{Conclusion/Future Studies}

Kalirin-7, the major isoform of Kalirin in the adult brain, plays a critical role in spine formation/synaptic plasticity. Estrogen-mediated spine formation in hippocampal neurons and cocaine-induced increases in spine density in striatal MSNs require Kalirin-7. Kalirin has also been implicated in many neuropsychiatric and neurological diseases. Future studies will focus on investigating the underlying molecular mechanisms of Kalirin-7-mediated spine formation/synaptic plasticity and its role in neuropsychiatric and neurological diseases.

\section{Acknowledgments}

This work was supported by grants DK32948, DA22876, MH086552, DA032973 from NIH and 11SCA28 from Connecticut Innovations. Thanks are due to Drs. Dick Mains and Betty Eipper for their comments.

\section{References}

[1] M. Rashidul Alam, B. D. Caldwell, R. G. Johnson, D. N. Darlington, R. E. Mains, and B. A. Eipper, "Novel proteins that interact with the $\mathrm{COOH}$-terminal cytosolic routing determinants of an integral membrane peptide-processing enzyme," Journal of Biological Chemistry, vol. 271, no. 45, pp. 28636-28640, 1996.

[2] M. R. Alam, R. C. Johnson, D. N. Darlington, T. A. Hand, R. E. Mains, and B. A. Eipper, "Kalirin, a cytosolic protein with spectrin-like and GDP/GTP exchange factor-like domains that interacts with peptidylglycine $\alpha$-amidating monooxygenase, an integral membrane peptide-processing enzyme," Journal of Biological Chemistry, vol. 272, no. 19, pp. 12667-12675, 1997.

[3] K. L. Rossman, C. J. Der, and J. Sondek, "GEF means go: turning on Rho GTPases with guanine nucleotide-exchange factors," Nature Reviews Molecular Cell Biology, vol. 6, no. 2, pp. 167-180, 2005.

[4] M. Negishi and H. Katoh, "Rho family GTPases and dendrite plasticity," Neuroscientist, vol. 11, no. 3, pp. 187-191, 2005.

[5] K. F. Tolias, J. G. Duman, and K. Um, "Control of synapse development and plasticity by Rho GTPase regulatory proteins," Progress in Neurobiology, vol. 94, no. 2, pp. 133-148, 2011.

[6] P. Penzes, K. M. Woolfrey, and D. P. Srivastava, "Epac2mediated dendritic spine remodeling: implications for disease," Molecular and Cellular Neuroscience, vol. 46, no. 2, pp. 368-380, 2011.

[7] R. C. Johnson, P. Penzes, B. A. Eipper, and R. E. Mains, "Isoforms of Kalirin, a neuronal Dbl family member, generated through use of different $5^{\prime}$ - and $3^{\prime}$-ends along with an internal translational initiation site," Journal of Biological Chemistry, vol. 275, no. 25, pp. 19324-19333, 2000.

[8] C. E. McPherson, B. A. Eipper, and R. E. Mains, "Genomic organization and differential expression of Kalirin isoforms," Gene, vol. 284, no. 1-2, pp. 41-51, 2002.

[9] C. E. McPherson, B. A. Eipper, and R. E. Mains, "Kalirin expression is regulated by multiple promoters," Journal of Molecular Neuroscience, vol. 22, no. 1-2, pp. 51-62, 2004.

[10] A. Hayashi-Takagi, M. Takaki, N. Graziane et al., "Disruptedin-Schizophrenia 1 (DISC1) regulates spines of the glutamate synapse via Rac1," Nature Neuroscience, vol. 13, no. 3, pp. 327$332,2010$.

[11] E. A. Ratovitski, M. R. Alam, R. A. Quick et al., "Kalirin inhibition of inducible nitric-oxide synthase," Journal of Biological Chemistry, vol. 274, no. 2, pp. 993-999, 1999.

[12] V. Colomer, S. Engelender, A. M. Sharp et al., "Huntingtinassociated protein 1 (HAP1) binds to a Trio-like polypeptide, with a racl guanine nucleotide exchange factor domain," Human Molecular Genetics, vol. 6, no. 9, pp. 1519-1525, 1997.

[13] T. H. Koo, B. A. Eipper, and J. G. Donaldson, "Arf6 recruits the Rac GEF Kalirin to the plasma membrane facilitating Rac activation,” BMC Cell Biology, vol. 8, article no. 29, 2007.

[14] C. A. Rabiner, R. E. Mains, and B. A. Eipper, "Kalirin: a dual Rho guanine nucleotide exchange factor that is so much more than the sum of its many parts," Neuroscientist, vol. 11, no. 2, pp. 148-160, 2005. 
[15] X. Xin, C. A. Rabiner, R. E. Mains, and B. A. Eipper, "Kalirin12 interacts with dynamin," BMC Neuroscience, vol. 10, article no. 61, 2009.

[16] P. Penzes, R. C. Johnson, V. Kambampati, R. E. Mains, and B. A. Eipper, "Distinct roles for the two Rho GDP/GTP exchange factor domains of Kalirin in regulation of neurite growth and neuronal morphology," Journal of Neuroscience, vol. 21, no. 21, pp. 8426-8434, 2001.

[17] M. R. Schiller, A. Blangy, J. Huang, R. E. Mains, and B. A. Eipper, "Induction of lamellipodia by Kalirin does not require its guanine nucleotide exchange factor activity," Experimental Cell Research, vol. 307, no. 2, pp. 402-417, 2005.

[18] T. Kawai, H. Sanjo, and S. Akira, "Duet is a novel serine/threonine kinase with Dbl-Homology (DH) and Pleckstrin-Homology (PH) domains," Gene, vol. 227, no. 2, pp. 249-255, 1999.

[19] X. M. Ma, Y. Wang, F. Ferraro, R. E. Mains, and B. A. Eipper, "Kalirin-7 is an essential component of both shaft and spine excitatory synapses in hippocampal interneurons," Journal of Neuroscience, vol. 28, no. 3, pp. 711-724, 2008.

[20] D. E. Hansel, M. E. Quiñones, G. V. Ronnett, and B. A. Eipper, "Kalirin, a GDP/GTP exchange factor of the Dbl family, is localized to nerve, muscle, and endocrine tissue during embryonic rat development," Journal of Histochemistry and Cytochemistry, vol. 49, no. 7, pp. 833-844, 2001.

[21] F. Ferraro, X. M. Ma, J. A. Sobota, B. A. Eipper, and R. E. Mains, "Kalirin/Trio Rho guanine nucleotide exchange factors regulate a novel step in secretory granule maturation," Molecular Biology of the Cell, vol. 18, no. 12, pp. 4813-4825, 2007.

[22] X. M. Ma, J. Huang, Y. Wang, B. A. Eipper, and R. E. Mains, "Kalirin, a multifunctional rho guanine nucleotide exchange factor, is necessary for maintenance of hippocampal pyramidal neuron dendrites and dendritic spines," Journal of Neuroscience, vol. 23, no. 33, pp. 10593-10603, 2003.

[23] D. Muller, P. A. Buchs, and L. Stoppini, "Time course of synaptic development in hippocampal organotypic cultures," Developmental Brain Research, vol. 71, no. 1, pp. 93-100, 1993.

[24] J. J. Hill, T. Hashimoto, and D. A. Lewis, "Molecular mechanisms contributing to dendritic spine alterations in the prefrontal cortex of subjects with schizophrenia," Molecular Psychiatry, vol. 11, no. 6, pp. 557-566, 2006.

[25] S. Narayan, B. Tang, S. R. Head et al., "Molecular profiles of schizophrenia in the CNS at different stages of illness," Brain Research, vol. 1239, pp. 235-248, 2008.

[26] I. Kushima, Y. Nakamura, B. Aleksic et al., "Resequencing and association analysis of the KALRN and EPHB1 genes and their contribution to Schizophrenia susceptibility," Schizophrenia Bulletin. In press.

[27] H. Youn, I. Ji, H. P. Ji, W. R. Markesbery, and T. H. Ji, "Underexpression of Kalirin-7 increases iNOS activity in cultured cells and correlates to elevated iNOS activity in Alzheimer's disease hippocampus," Journal of Alzheimer's Disease, vol. 12, no. 3, pp. 271-281, 2007.

[28] H. Youn, M. Jeoung, Y. Koo et al., "Kalirin is under-expressed in Alzheimer's disease hippocampus," Journal of Alzheimer's Disease, vol. 11, no. 3, pp. 385-397, 2007.

[29] A. Alttoa, K. Kõiv, T. A. Hinsley, A. Brass, and J. Harro, "Differential gene expression in a rat model of depression based on persistent differences in exploratory activity," European Neuropsychopharmacology, vol. 20, no. 5, pp. 288-300, 2010.

[30] X. M. Ma, R. E. Mains, and B. A. Eipper, "Plasticity in hippocampal peptidergic systems induced by repeated electroconvulsive shock," Neuropsychopharmacology, vol. 27, no. 1, pp. 55-71, 2002.
[31] W. Li, Q. J. Li, and S. C. An, "Preventive effect of estrogen on depression-like behavior induced by chronic restraint stress," Neuroscience Bulletin, vol. 26, no. 2, pp. 140-146, 2010.

[32] A. K. Sharma, G. H. Searfoss, R. Y. Reams et al., "Kainic acidinduced f-344 rat model of Mesial temporal lobe epilepsy: gene expression and canonical Pathways," Toxicologic Pathology, vol. 37, no. 6, pp. 776-789, 2009.

[33] K.-P. Lesch, N. Timmesfeld, T. J. Renner et al., "Molecular genetics of adult ADHD: converging evidence from genomewide association and extended pedigree linkage studies," Journal of Neural Transmission, vol. 115, no. 11, pp. 1573-1585, 2008.

[34] M. Beręsewicz, J. E. Kowalczyk, and B. Zabłocka, "Kalirin7, a protein enriched in postsynaptic density, is involved in ischemic signal transduction," Neurochemical Research, vol. 33, no. 9, pp. 1789-1794, 2008.

[35] T. Krug, H. Manso, L. Gouveia et al., "Kalirin: a novel genetic risk factor for ischemic stroke," Human Genetics, vol. 127, no. 5, pp. 513-523, 2010.

[36] L. Wang, E. R. Hauser, S. H. Shah et al., "Peakwide mapping on chromosome $3 \mathrm{q} 13$ identifies the kalirin gene as a novel candidate gene for coronary artery disease," American Journal of Human Genetics, vol. 80, no. 4, pp. 650-663, 2007.

[37] R. E. Mains, D. D. Kiraly, J. E. Eipper-Mains, X. Ma, and B. A. Eipper, "Kalrn promoter usage and isoform expression respond to chronic cocaine exposure," BMC Neuroscience, vol. 12, article 20, 2011.

[38] D. D. Kiraly, X. M. Ma, C. M. Mazzone, X. Xin, R. E. Mains, and B. A. Eipper, "Behavioral and morphological responses to cocaine require Kalirin7,” Biological Psychiatry, vol. 68, no. 3, pp. 249-255, 2010.

[39] P. Penzes, R. C. Johnson, R. Sattler et al., "The neuronal RhoGEF Kalirin-7 interacts with PDZ domain-containing proteins and regulates dendritic morphogenesis," Neuron, vol. 29, no. 1, pp. 229-242, 2001.

[40] X.-M. Ma, J.-P. Huang, E.-J. Kim et al., "Kalirin-7, an important component of excitatory synapses, is regulated by estradiol in hippocampal neurons," Hippocampus, vol. 21, no. 6, pp. 661-677, 2011.

[41] D. D. Kiraly, F. Lemtiri-Chlieh, E. S. Levine, R. E. Mains, and B. A. Eipper, "Kalirin binds the NR2B subunit of the NMDA receptor, altering its synaptic localization and function," Journal of Neuroscience, vol. 31, no. 35, pp. 12554-12565, 2011.

[42] Z. Xie, D. P. Srivastava, H. Photowala et al., "Kalirin-7 controls activity-dependent structural and functional plasticity of dendritic spines," Neuron, vol. 56, no. 4, pp. 640-656, 2007.

[43] X. Xin, Y. Wang, X. M. Ma et al., "Regulation of Kalirin by Cdk5," Journal of Cell Science, vol. 121, no. 15, pp. 2601-2611, 2008.

[44] M. E. Cahill, K. A. Jones, I. Rafalovich et al., "Control of interneuron dendritic growth through NRG1/erbB4-mediated kalirin-7 disinhibition," Molecular Psychiatry, vol. 17, no. 1, pp. 99-107, 2012.

[45] D. D. Kiraly, K. L. Stone, C. M. Colangelo et al., "Identification of kalirin-7 as a potential post-synaptic density signaling hub," Journal of Proteome Research, vol. 10, no. 6, pp. 2828-2841, 2011.

[46] X. M. Ma, R. C. Johnson, R. E. Mains, and B. A. Eipper, "Expression of Kalirin, a neuronal GDP/GTP exchange factor of the trio family, in the central nervous system of the adult rat," Journal of Comparative Neurology, vol. 429, no. 3, pp. 388402, 2001.

[47] R. T. Fremeau, M. D. Troyer, I. Pahner et al., "The expression of vesicular glutamate transporters defines two classes of excitatory synapse," Neuron, vol. 31, no. 2, pp. 247-260, 2001. 
[48] R. T. Fremeau, K. Kam, Y. Qureshi et al., "Vesicular glutamate transporters 1 and 2 target to functionally distinct synaptic release sites," Science, vol. 304, no. 5678, pp. 1815-1819, 2004.

[49] G. Maccaferri and J. C. Lacaille, "Interneuron Diversity series: hippocampal interneuron classifications-making things as simple as possible, not simpler," Trends in Neurosciences, vol. 26, no. 10, pp. 564-571, 2003.

[50] F. M. Benes and S. Berretta, "GABAergic interneurons: implications for understanding schizophrenia and bipolar disorder," Neuropsychopharmacology, vol. 25, no. 1, pp. 1-27, 2001.

[51] E. M. Powell, D. B. Campbell, G. D. Stanwood, C. Davis, J. L. Noebels, and P. Levitt, "Genetic disruption of cortical interneuron development causes region- and GABA cell typespecific deficits, epilepsy, and behavioral dysfunction," Journal of Neuroscience, vol. 23, no. 2, pp. 622-631, 2003.

[52] D. A. Lewis, T. Hashimoto, and D. W. Volk, "Cortical inhibitory neurons and schizophrenia," Nature Reviews Neuroscience, vol. 6, no. 4, pp. 312-324, 2005.

[53] B. Ramos, D. Baglietto-Vargas, J. C. D. Rio et al., "Early neuropathology of somatostatin/NPY GABAergic cells in the hippocampus of a PS1 $\times$ APP transgenic model of Alzheimer's disease," Neurobiology of Aging, vol. 27, no. 11, pp. 1658-1672, 2006.

[54] X. M. Ma, D. D. Kiraly, E. D. Gaier et al., "Kalirin-7 is required for synaptic structure and function," Journal of Neuroscience, vol. 28, no. 47, pp. 12368-12382, 2008.

[55] M. E. Cahill, Z. Xie, M. Day et al., "Kalirin regulates cortical spine morphogenesis and disease-related behavioral phenotypes," Proceedings of the National Academy of Sciences of the United States of America, vol. 106, no. 31, pp. 13058-13063, 2009.

[56] K. O. Lai and N. Y. Ip, "Recent advances in understanding the roles of Cdk5 in synaptic plasticity," Biochimica et Biophysica Acta, vol. 1792, no. 8, pp. 741-745, 2009.

[57] K. Akashi, T. Kakizaki, H. Kamiya et al., "NMDA receptor GluN2B (GluRe2/NR2B) subunit is crucial for channel function, postsynaptic macromolecular organization, and actin cytoskeleton at hippocampal CA3 synapses," Journal of Neuroscience, vol. 29, no. 35, pp. 10869-10882, 2009.

[58] J. L. Brigman, T. Wright, G. Talani et al., "Loss of GluN2Bcontaining NMD A receptors in CA1 hippocampus and cortex impairs long-term depression, reduces dendritic spine density, and disrupts learning," Journal of Neuroscience, vol. 30, no. 13, pp. 4590-4600, 2010.

[59] T. Ohno, H. Maeda, N. Murabe et al., "Specific involvement of postsynaptic GluN2B-containing NMDA receptors in the developmental elimination of corticospinal synapses," Proceedings of the National Academy of Sciences of the United States of America, vol. 107, no. 34, pp. 15252-15257, 2010.

[60] A. C. Gambrill and A. Barria, "NMDA receptor subunit composition controls synaptogenesis and synapse stabilization," Proceedings of the National Academy of Sciences of the United States of America, vol. 108, no. 14, pp. 5855-5860, 2011.

[61] Z. Xie, M. E. Cahill, J. Radulovic et al., "Hippocampal phenotypes in kalirin-deficient mice," Molecular and Cellular Neuroscience, vol. 46, no. 1, pp. 45-54, 2011.

[62] J. Martínez-Raga, C. Knecht, and S. Cepeda, "Modafinil: a useful medication for cocaine addiction? Review of the evidence from neuropharmacological, experimental and clinical studies," Current drug abuse reviews, vol. 1, no. 2, pp. 213-221, 2008.

[63] M. J. Smith, J. Thirthalli, A. B. Abdallah, R. M. Murray, and L. B. Cottler, "Prevalence of psychotic symptoms in substance users: a comparison across substances," Comprehensive Psychiatry, vol. 50, no. 3, pp. 245-250, 2009.

[64] M. D. Majewska, "Cocaine addiction as a neurological disorder: implications for treatment," NIDA research monograph, vol. 163, pp. 1-26, 1996.

[65] J. H. Mendelson and N. K. Mello, "Drug therapy: management of cocaine abuse and dependence," New England Journal of Medicine, vol. 334, no. 15, pp. 965-972, 1996.

[66] A. Büttner, "Review: the neuropathology of drug abuse," Neuropathology and Applied Neurobiology, vol. 37, no. 2, pp. 118-134, 2011.

[67] D. D. Kiraly, X. M. Ma, C. M. Mazzone, X. Xin, R. E. Mains, and B. A. Eipper, "Behavioral and morphological responses to cocaine require Kalirin7," Biological Psychiatry, vol. 68, no. 3, pp. 249-255, 2010.

[68] J. K. Millar, S. Christie, and D. J. Porteous, "Yeast two-hybrid screens implicate DISC1 in brain development and function," Biochemical and Biophysical Research Communications, vol. 311, no. 4, pp. 1019-1025, 2003.

[69] N. J. Bradshaw and D. J. Porteous, "DISC1-binding proteins in neural development, signalling and schizophrenia," Neuropharmacology, vol. 62, no. 3, pp. 1230-1241, 2012.

[70] M. L. Berlanga, D. L. Price, B. S. Phung et al., "Multiscale imaging characterization of dopamine transporter knockout mice reveals regional alterations in spine density of medium spiny neurons," Brain Research, vol. 1390, pp. 41-49, 2011.

[71] V. Jensen, J. E. Rinholm, T. J. Johansen et al., "N-methyl$\mathrm{d}$-aspartate receptor subunit dysfunction at hippocampal glutamatergic synapses in an animal model of attentiondeficit/hyperactivity disorder," Neuroscience, vol. 158, no. 1, pp. 353-364, 2009.

[72] C. Tackenberg, A. Ghori, and R. Brandt, "Thin, stubby or mushroom: spine pathology in alzheimer's disease," Current Alzheimer Research, vol. 6, no. 3, pp. 261-268, 2009.

[73] S. J. Baloyannis, "Dendritic pathology in Alzheimer's disease," Journal of the Neurological Sciences, vol. 283, no. 1-2, pp. 153157, 2009.

[74] I. A. Mavroudis, D. F. Fotiou, M. G. Manani et al., "Dendritic pathology and spinal loss in the visual cortex in Alzheimer's disease: a Golgi study in pathology," International Journal of Neuroscience, vol. 121, no. 7, pp. 347-354, 2011.

[75] C. Perez-Cruz, M. W. Nolte, M. M. Van Gaalen et al., "Reduced spine density in specific regions of CA1 pyramidal neurons in two transgenic mouse models of Alzheimer's disease," Journal of Neuroscience, vol. 31, no. 10, pp. 3926-3934, 2011.

[76] O. von Bohlen und Halbach, "Structure and function of dendritic spines within the hippocampus," Annals of Anatomy, vol. 191, no. 6, pp. 518-531, 2009.

[77] A. J. Law, C. S. Weickert, T. M. Hyde, J. E. Kleinman, and P. J. Harrison, "Reduced spinophilin but not microtubuleassociated protein 2 expression in the hippocampal formation in schizophrenia and mood disorders: molecular evidence for a pathology of dendritic spines," American Journal of Psychiatry, vol. 161, no. 10, pp. 1848-1855, 2004.

[78] S. D. Norrholm and C. C. Ouimet, "Altered dendritic spine density in animal models of depression and in response to antidepressant treatment," Synapse, vol. 42, no. 3, pp. 151-163, 2001.

[79] F. Chen, T. M. Madsen, G. Wegener, and J. R. Nyengaard, "Repeated electroconvulsive seizures increase the total number of synapses in adult male rat hippocampus," European Neuropsychopharmacology, vol. 19, no. 5, pp. 329-338, 2009.

[80] M. Fink, "Convulsive therapy: a review of the first 55 years," Journal of Affective Disorders, vol. 63, no. 1-3, pp. 1-15, 2001. 
[81] S. M. McClintock, A. R. Brandon, M. M. Husain, and R. B. Jarrett, "A systematic review of the combined use of electroconvulsive therapy and psychotherapy for depression," The Journal of ECT, vol. 27, pp. 236-243, 2011.

[82] J. C. Fiala, J. Spacek, and K. M. Harris, "Dendritic spine pathology: cause or consequence of neurological disorders?" Brain Research Reviews, vol. 39, no. 1, pp. 29-54, 2002.

[83] C. Sgobio, V. Ghiglieri, C. Costa et al., "Hippocampal synaptic plasticity, memory, and epilepsy: effects of long-term valproic acid treatment," Biological Psychiatry, vol. 67, no. 6, pp. 567$574,2010$.

[84] L. L. Y. Wu and X. F. Zhou, "Huntingtin associated protein 1 and its functions," Cell Adhesion and Migration, vol. 3, no. 1, pp. 71-76, 2009.

[85] J.-P. Vonsattel, R. H. Myers, and T. J. Stevens, "Neuropathological classification of Huntington's disease," Journal of Neuropathology and Experimental Neurology, vol. 44, no. 6, pp. 559-577, 1985.

[86] G. A. Graveland, R. S. Williams, and M. DiFiglia, "Evidence for degenerative and regenerative changes in neostriatal spiny neurons in Huntington's disease," Science, vol. 227, no. 4688, pp. 770-773, 1985.

[87] R. J. Ferrante, N. W. Kowall, and E. P. Richardson, "Proliferative and degenerative changes in striatal spiny neurons in Huntington's disease: a combined study using the sectionGolgi method and calbindin D28k immunocytochemistry," Journal of Neuroscience, vol. 11, no. 12, pp. 3877-3887, 1991.

[88] T. L. Spires, H. E. Grote, S. Garry et al., "Dendritic spine pathology and deficits in experience-dependent dendritic plasticity in R6/1 Huntington's disease transgenic mice," European Journal of Neuroscience, vol. 19, no. 10, pp. 27992807, 2004.

[89] M. I. Boulware, B. A. Kent, and K. M. Frick, "The impact of age-related ovarian hormone loss on cognitive and neural function," Current Topics in Behavioral Neurosciences. In press.

[90] E. Scott, Q.-G. Zhang, R. Wang, R. Vadlamudi, and D. Brann, "Estrogen neuroprotection and the critical period hypothesis," Frontiers in Neuroendocrinology, vol. 33, no. 1, pp. 85-104, 2012.

[91] A. Riecher-Rössler and J. Kulkarni, "Estrogens and gonadal function in schizophrenia and related psychoses," Current topics in behavioral neurosciences, vol. 8, pp. 155-171, 2011. 

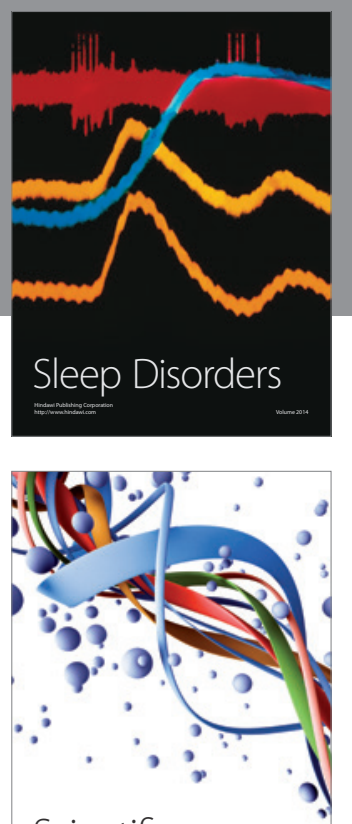

Scientifica
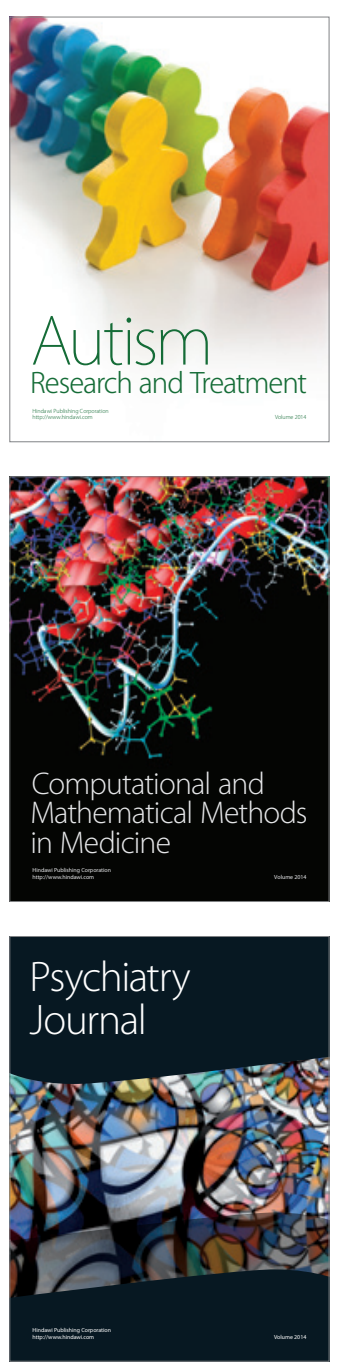
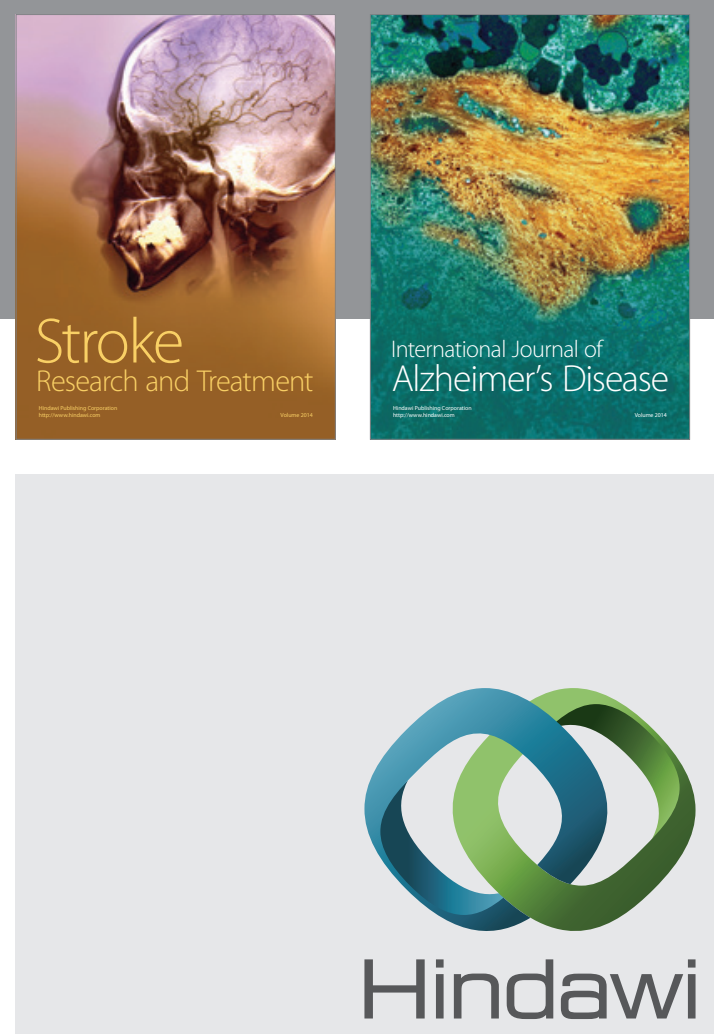

Submit your manuscripts at

http://www.hindawi.com
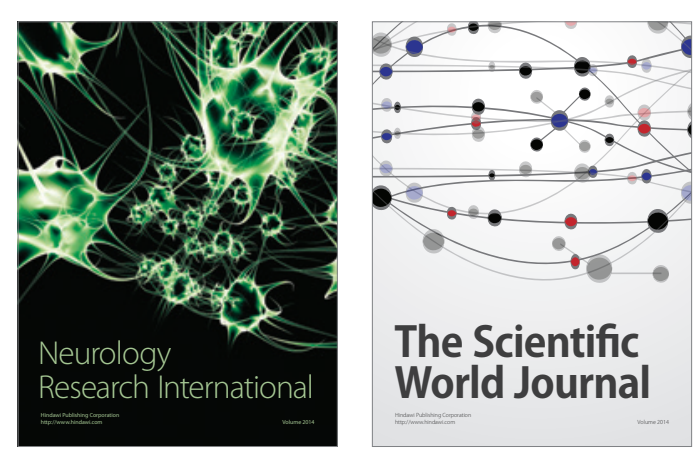

The Scientific World Journal

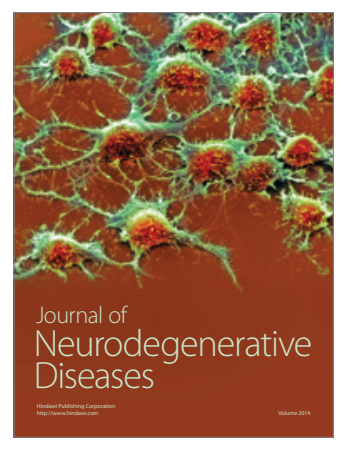

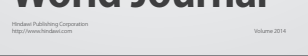

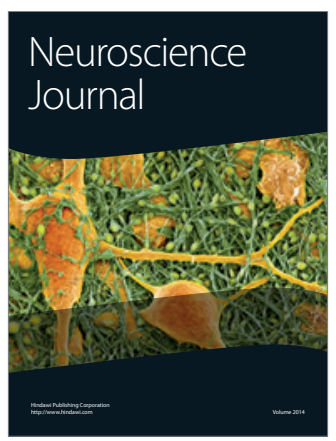

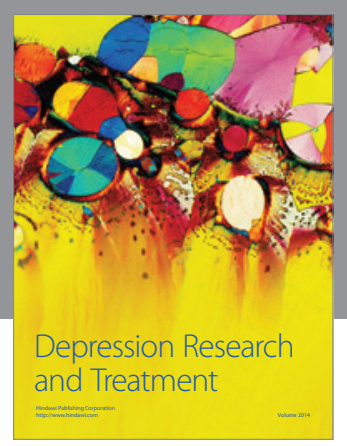
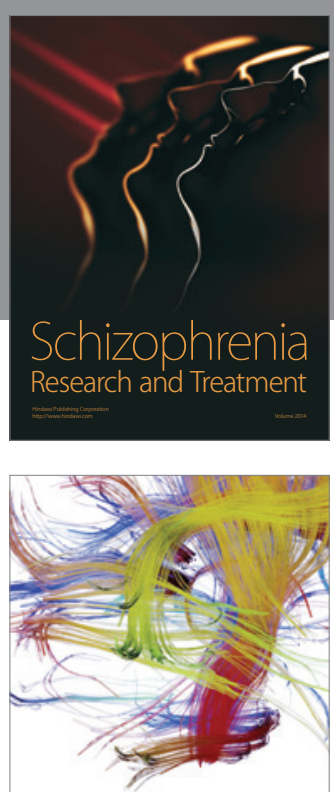

Brain Science

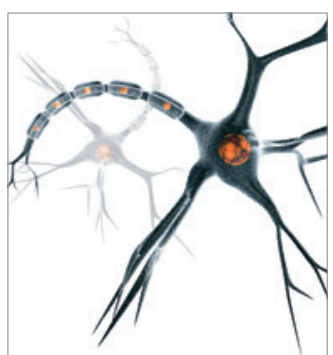

Neural Plasticity
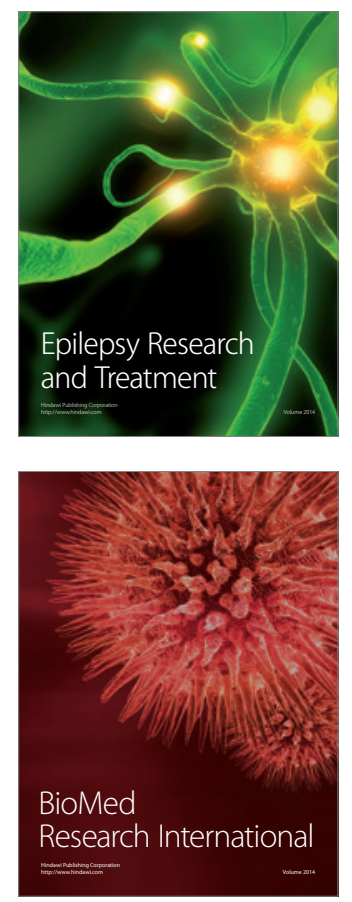

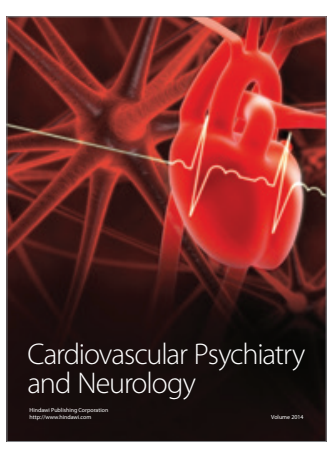

Parkinson's

Disease
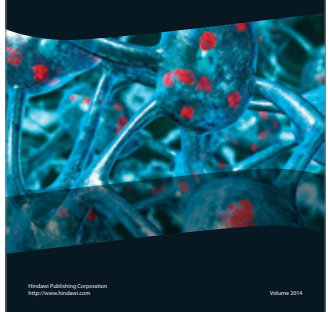